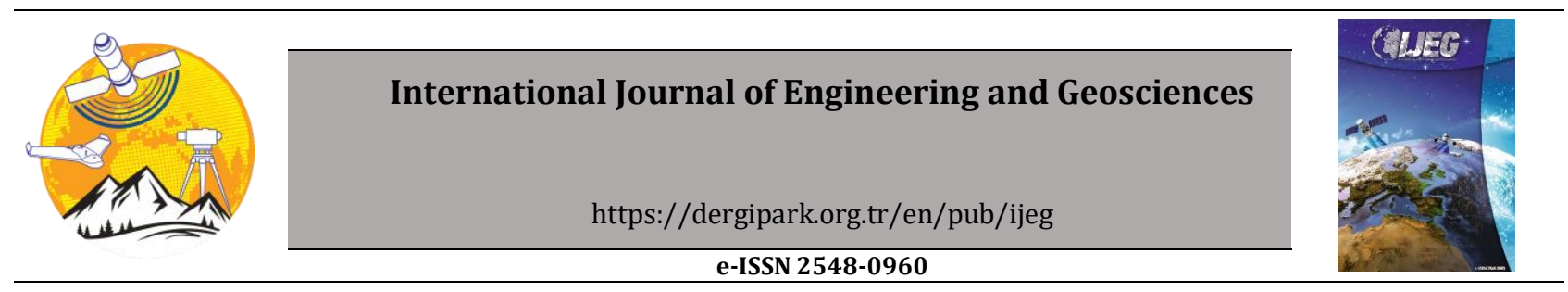

\title{
An investigation of the performances of polarimetric target decompositions using GB- SAR imaging
}

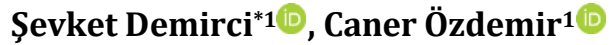 \\ 1Mersin University, Engineering Faculty, Electrical and Electronics Engineering Department, Mersin, Turkey
}

\author{
Keywords \\ SAR \\ Polarimetric SAR \\ Ground-based SAR \\ Target decomposition
}

\begin{abstract}
Ground-based synthetic aperture radar (GB-SAR) systems are mostly utilized to be practical practices in improved understanding of the complex mechanism of microwave backscattering. They also provide complementary information on evaluating the validity of the polarimetric analysis of air-borne or satellite-borne SAR applications. This study investigates some capabilities of polarimetric L-band GB-SAR imaging by testing its performance against a typical terrain and various kinds of manmade targets. Trihedral corner reflectors are also included in the analyses because of their importance in data calibration. Polarimetric backscattering signatures of different targets are analyzed in terms of qualitative assessment of amplitude images and identification and classification of scattering mechanisms through target decomposition techniques. The findings of these analyses and detailed discussions are presented. Specifically, the entropy/meanalpha $((H / \bar{\alpha}))$ classification results are shown to be capable of clearly identifying the dominant scattering mechanisms occurring within the investigated scene.
\end{abstract}

\section{INTRODUCTION}

Active remote sensors supply their own illumination energy to acquire information about the Earth's surface. RADAR (Radio Detection and Ranging) and LIDAR (Light Detection and Ranging) are two typical examples of active sensors which have been widely used in a variety of environmental applications, such as classification of urban and forest areas and production of digital elevation models (DEM) (Moreira et al. 2013; Sevgen 2019; Yilmaz and Uysal 2017; Yilmaz and Erdogan 2018).

Among the radar sensors, synthetic aperture radar (SAR) constitutes one of the essential technologies for microwave remote sensing. Basically, it uses the motion of the platform on which the radar is mounted to generate an image of the Earth's surface. SAR systems operating on aircrafts provide monitoring of large-scale areas with relatively high resolution imagery (Ouchi 2013). On the other hand, ground-based SAR (GB-SAR) systems are better suited for inspection of small-scale areas with a better resolution capability than that of air- based systems (Cho et al. 2006; Gonzalez et al. 2008; Lee et al. 2007; Lee et al. 2014; Lee et al. 2016; Penner and Long 2017; Cuenca 2017). Interferometric and polarimetric practices can also be effectively exploited within these systems to aid target identification. This type of ground-truth information could also be helpful for research and validation studies. Herein, we carry out such a study by focusing on the assessment of polarimetric GBSAR imaging of a typical land scene.

In polarimetric SAR (PolSAR), data are collected with different combinations of transmit-receive polarizations. It has well known that radar polarimetry is sensitive to the structure of the target being observed. In principle, the target's reflectivity, shape, symmetric structures can be extracted from the reflected wave's polarization change data. This is, however, a difficult task, since the interaction between microwaves and random media is usually complex, varying as a function of numerous factors such as wavelength, viewing geometry, polarization of the transmitted wave as well as target attributes including roughness, shape, size and orientation. In target decompositions using GB-SAR imaging. International Journal of Engineering and Geosciences, 6(1), 09-19 
past decades, a great amount of effort has been made to overcome this limitation (Lee and Pottier 2009; van Zyl and Kim 2011; Cloude 2010). This has given rise to development of various polarimetric SAR (PolSAR) systems and methods which have proven the usefulness of PolSAR in various applications especially in image classification and contrast enhancement. Among these, for GB-SAR specific applications, the reader is referred to (Pipia 2009; Pipia et al. 2013; Iglesias et al. 2015a; Iglesias et al. 2015b; Baffelli et al. 2018; Brown et al. 2003; Lim and Koo 2008; Penner and Long 2017; Zhou 2003; Zhou 2004; Minh et al. 2014; Kang et al. 2009; Xing et al. 2013). Nevertheless, it is still an experimental technique in quantitative retrieval of target parameters.

In this context, polarimetric target decomposition techniques remain the most promising tools (Chen et al. 2018). Such techniques decompose the measured backscattering matrix into a combination of simpler (canonical) responses. This provides a better identification of scattering mechanisms and thus an easier interpretation of targets' structural characteristics. To date, many successful applications of several decomposition approaches have been reported for a variety of SAR data (Lee and Pottier 2009; Chen et al. 2018; Chen et al. 2014; Alberga et al. 2004; Cloude 2010). However, it is also obvious that the wide variability and complexity in target scenes and environmental conditions may lead to misinterpretation if groundtruth information is not available. Consequently, the performances of these techniques might be anecdotal, with good performance under certain conditions and poor performance elsewhere.

In our previous study (Demirci et al. 2019), we therefore, tested the potential of the two of the most widely used techniques namely; Pauli and eigenvalue/eigenvector decomposition with data from a fully-controlled GB-SAR experiment. In this paper, we extend this analysis by including Krogager decomposition (Krogager 1990) and FreemanDurden decomposition (Freeman and Durden 1998), so that comparisons can be made. Also, a different land scene consisting of a series of trees is employed because of the growing interest in vegetation monitoring (Penner and Long 2017; Zhou 2004; Minh et al. 2014; Albinet et al. 2012). The other investigated targets are; a gazebo with waste containers and metal sticks and corner reflectors added on the surface. The relevant images of the mentioned decompositions at L-band are analyzed to recognize the targets' identifiable features over image pixels. The findings of these analyses and discussions are presented.

\section{THEORY}

\subsection{GB-SAR Imaging Methodology}

Fig. 1 shows the geometry for a typical twodimensional (2D) monostatic GB-SAR imaging.
Monostatic means the same antenna is used as the transmitter (TX) and receiver (RX). The antenna is elevated to a certain height $h$ and inclined with an incidence angle $\beta^{\prime}$ from the vertical direction. With transmission of a single pulse, the scene is illuminated with a range of local incidence angles $\beta$. The radar records part of the scattered wave directed backwards to the RX antenna. The process is then repeated by measuring the returned signals at $\Delta x$ azimuth steps and covering a total synthetic aperture length $L_{\text {synt }}$. If the reflectivity of the desired imaging area is denoted by $D(x, y)$, then $y$ is normal to the scanning path corresponding to ground-range axis, while $x$ is parallel to the platform path corresponding to azimuth or cross-range dimension. The range or slant-range $r$ refers to the true distance from antenna to target.

Assuming a stepped-frequency continuous wave (SFCW) transmission, the received signal at a specific scanning point contains the frequency response of the scene, sampled at discrete frequency steps $\Delta f$ within a bandwidth $B$. The inverse Fourier transform (IFT) of this signal provides the range profile of the illuminated area for that particular viewpoint. The range profile represents a projection of a three-dimensional (3D) target into a onedimensional (1D) function. Obtaining an estimate of target reflectivity (i.e., imaging) can then be thought as a process of inverting this transform. For such near-field data collections, plane-wave illumination of the entire target is usually not satisfied, thus an image reconstruction algorithm that accounts for the wavefront curvature effects is needed to obtain a focused image.

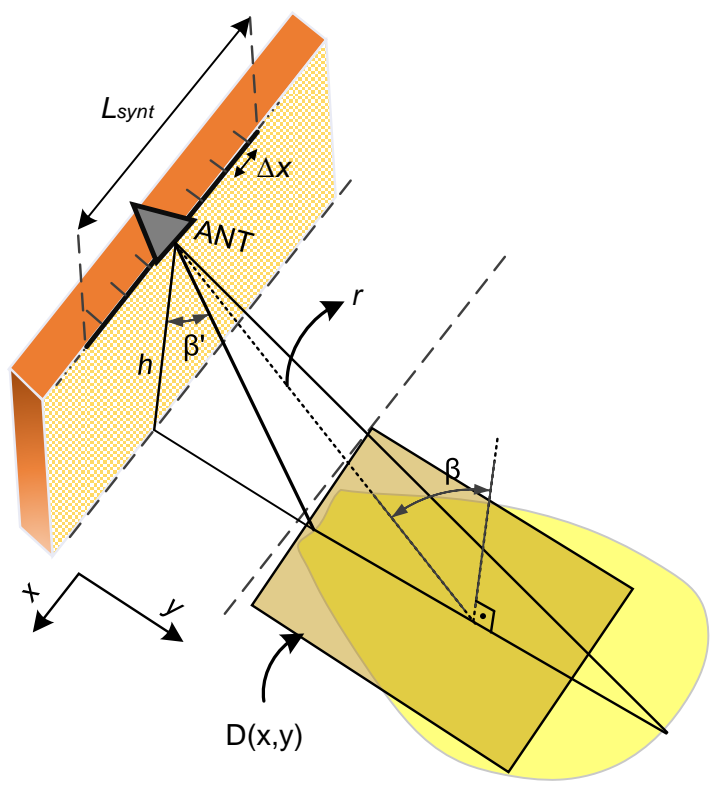

Figure 1. The geometry for 2D monostatic GB-SAR imaging

\subsection{PolSAR Data Characteristics}

PolSAR systems operate mostly in linear horizontal $(\mathrm{H})$ and vertical $(\mathrm{V})$ polarization basis, 
measuring up to four channels, i.e., $H H, V V, H V$ and $V H$ with the first and second letters represent transmit and receive polarizations, respectively. The backscattering characteristics of a target can be completely described by a $2 \times 2$ scattering (Sinclair) matrix $[S]$

$$
[S]=\left[\begin{array}{ll}
S_{H H} & S_{H V} \\
S_{V H} & S_{V V}
\end{array}\right]
$$

where the elements are the complex scattering amplitudes measured by the corresponding channel of radar. The diagonal and off-diagonal elements are termed as co-pol and cross-pol channels, respectively. In monostatic radars, $[S]$ becomes symmetric, i.e., $S_{H V}=S_{V H}=S_{X X}$ for targets that reciprocity property holds.

Each pixel in a focused SAR image retains this single complex dataset which is dependent only on the target parameters for a fixed viewing geometry and frequency. Many targets of interest in SAR imaging can be broadly categorized into two: deterministic (coherent) and distributed (incoherent). Deterministic targets lead to nondepolarizing scattering process that can well be described by a first-order descriptor, such as the $[S]$ matrix. Distributed targets, on the other hand, normally give rise to either partially or completely depolarizing scattering process owing to presence of many randomly distributed point scatterers inside a resolution unit. In such cases, second order descriptors, such as coherency $[T]$ and covariance $[C]$ matrices are utilized to describe the scattering behavior of each pixel, together with a spatial averaging over adjacent pixels.

\subsection{Target Decomposition Techniques}

Polarimetric target decomposition techniques allow interpretation of the measured polarimetric radar data by separating it into basic (canonical) scattering mechanisms. There are numerous decomposition schemes which mainly fall into two types: coherent decomposition and incoherent decomposition. The former is based on the decomposition of $[S]$ matrix whereas the latter is based on the decomposition of $[T]$ or $[C]$ matrices. Among the popular coherent decompositions are the Pauli decomposition (Lee and Pottier 2009) and the Krogager decomposition (Krogager 1990). As for the incoherent decomposition, it is further rendered into two classes: eigenvector/eigenvalue based decomposition (Cloude and Pottier 1996; Cloude and Pottier 1997) and model-based decomposition (Chen et. al. 2014). The first is based on the eigenvalue analysis of the [T] matrix and has the capability of incorporating the entire range of scattering mechanisms. The parameters, namely entropy $(H)$ and mean-alpha $(\bar{\alpha})$ derived from this decomposition can also be utilized within unsupervised classification algorithms, like the wellknown $\mathrm{H} / \bar{\alpha}$ classification (Cloude and Pottier 1997).
The second class of incoherent decomposition makes use of various scattering models to decompose the scattering power contained in $[T]$ or $[C]$ matrix. The most typical example is the Freeman-Durden decomposition (Freeman and Durden 1998) which is also the first developed model-based decomposition technique. For a detailed discussion of target decomposition principles and applications, the reader is referred to (Lee and Pottier 2009; Chen et al. 2018).

\section{MEASUREMENT SETUP and TEST SCENE}

To perform stripmap SAR measurements, we constructed a radar system and mounted it onto a wheel platform. The system consists of a vector network analyzer (VNA) that works as a SFCW radar between $0.3 \mathrm{GHz}$ and $8.5 \mathrm{GHz}$, two Vivaldi type horn antennas in a quasi-monostatic arrangement, a 1 Watt RF amplifier and a computer with a MATLAB [The Mathworks] program that controls and synchronizes positioning and data capturing. The complex data measured for a single-sweep of VNA can be instantaneously imported into MATLAB for post-processing.

With the geometry depicted in Fig. 1, a field experiment was conducted. A terrain near a building in Mersin University was selected and the measurements were carried out on that said building's roof terrace at a height of $15 \mathrm{~m}$ above ground. Fig. 2 shows the picture of the investigated scene seen from the radar location. The scene was comprised of a soil surface partly covered with grasses and bushes, a series of trees, a pave road and a gazebo. We also added various metallic objects with different orientations onto surface to determine and validate canonical backscattering mechanisms. The zoomed-out views of these objects, namely; five trihedral corner reflectors ( $\mathrm{C} 1$ to $\mathrm{C} 5$ ) and four metal sticks (T1 to T4) can be seen in the upper pictures of Fig. 2. C1 to C4 were of triangular type while C5 was of a square type. Trihedral C3 was put within trees and not optically visible throughout the synthetic aperture scan. As for the metal sticks, T1 was aligned horizontally while $\mathrm{T} 2$ vertically. The others $\mathrm{T} 3$ and T4 were oriented at about $-45^{\circ}$ and $45^{\circ}$ with respect to line-of-sight (LOS) direction, respectively.

The measurements were made for a frequency span of 1 to $3 \mathrm{GHz}$ sampled at 801 points and a synthetic aperture length of $L_{\text {synt }}=30 \mathrm{~m}$ sampled with $\Delta x=10 \mathrm{~cm}$ steps. The TX and RX antennas were both inclined to have an elevation angle of $81^{\circ}$ and operated in quasi-monostatic mode with a spatial separation of $30 \mathrm{~cm}$ between each other. In such quasi-monostatic backscattering measurements, reciprocity holds for most targets, whereby cross-pol terms are assumed to be equal, i.e., $S_{H V}=S_{V H}$. Therefore, we collected data in three channels, i.e., $S_{H H}, S_{V V}$ and $S_{V H}$. The following processing steps were taken in imaging: First, windowing with a Hanning smoothing function and a subsequent $4 \times$ zero padding were applied to the 
1D frequency-domain data measured for each sweep of VNA. Second, a 1D IFT was performed to obtain the corresponding range profiles. Finally, the resulted signals were focused via backprojection imaging algorithm, an explanation of which can be found in (Ozdemir et al. 2014) for a similar SAR geometry.

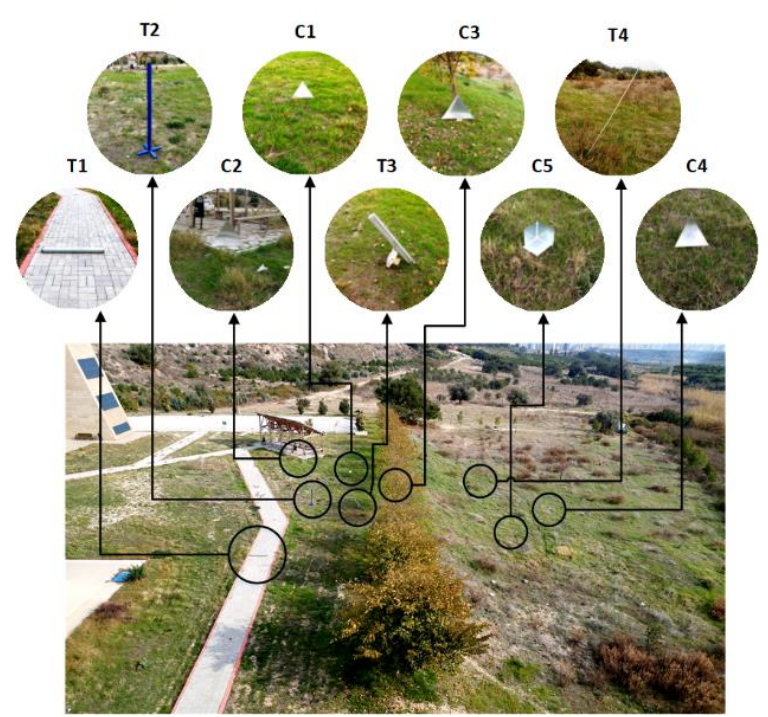

Figure 2. Picture of the scene seen from the radar location (down) and zoomed-out views of the manmade targets added on the scene (up)

\section{RESULTS}

\subsection{Amplitude Images}

Fig. 3 shows the reconstructed images in amplitude domain. Canonical objects are marked on the $\left|S_{V V}\right|$ image with squares. Note that, the layout of the terrain and its different sections can be inferred from each image. Many comments can be derived. To begin with let's first consider the $\left|S_{V H}\right|$ result. Theoretically, cross-pol scattering is mostly caused by natural media, complex manmade targets as well as oriented objects. The measured $\left|S_{V H}\right|$ image manifests this fact, since the targets that have complex structures like the trees, bushes, gazebo as well as the tilted sticks T3 and T4 are seen to have cross-pol component because of volume scattering mechanism. Besides, $V H$ scattering from the vertical cylinder T2, which is not expected, can be attributed to its complex-shaped base, which can be noticed from Fig. 2. Cross-pol amplitudes of the other objects are mostly low and not enough to produce a good contrast level.

Next, examining the co-pol signatures, we find it in the first place that all targets, either natural or manmade, are clearly displayed in each case, with $\left|S_{H H}\right|$ amplitudes being slightly higher than those of $\left|S_{V V}\right|$. Manmade targets, as expected, show strong non-depolarizing scattering and thus are well localized. Even the trihedral C3 within trees can be detected thanks to the capability of L-band electromagnetic waves in penetrating through tree canopies. As for the terrain targets, co-pol reflection from trees are shown to have larger amplitudes when compared to cross-pol case. This indicates dominance of odd or even-bounce scattering mechanisms produced from tree tops and trunkground interactions. The relatively larger grasses and bushes spread over the area also possess considerable co-pol scattering due to quasi-specular reflections. This is especially visible in $\left|S_{H H}\right|$ image wherein various patches of vegetation can be identified.
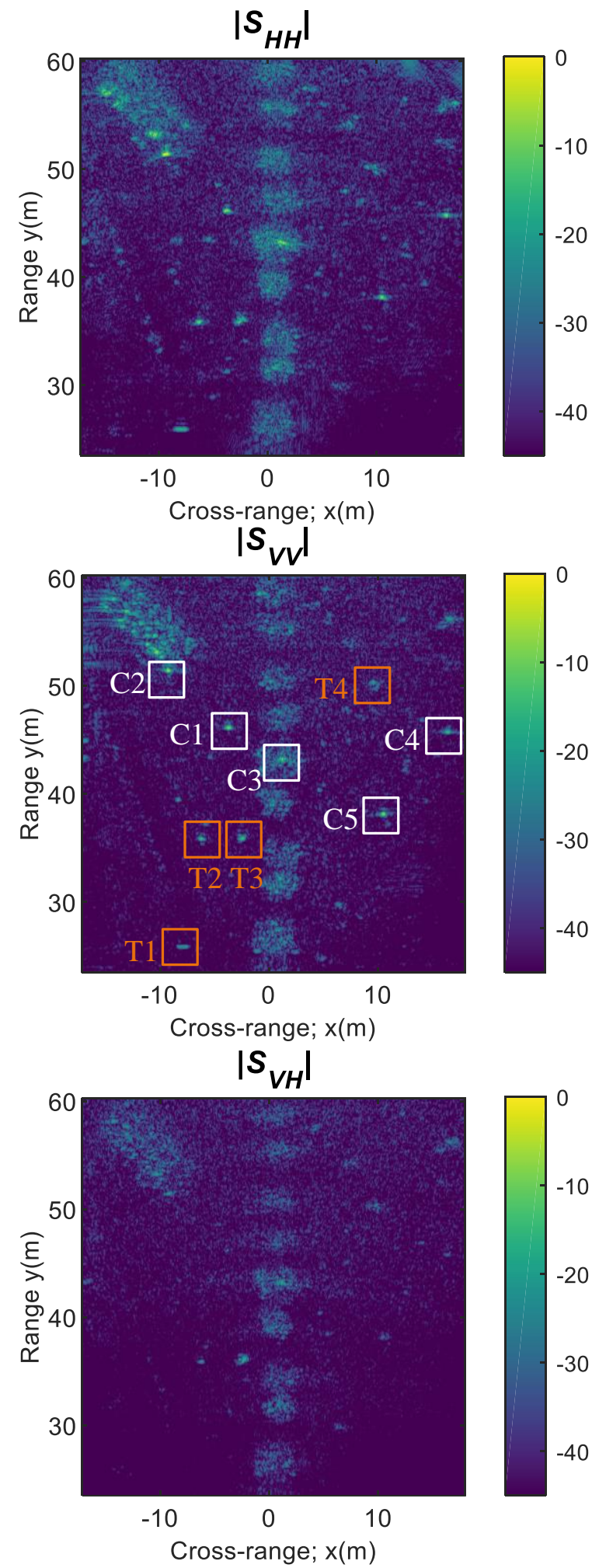

Figure 3. Amplitude images of the scattering matrix elements in a $\mathrm{dB}$ scale. Locations of the canonical objects are marked on the $\left|S_{V V}\right|$ image 


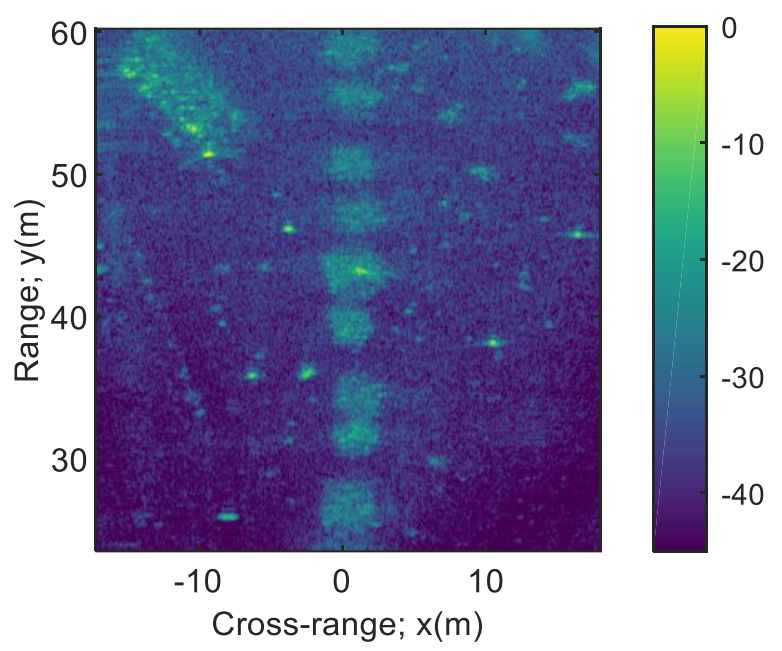

Figure 4. Total power image of the scattering matrix elements in a dB scale

For a power domain analysis, the total backscattered power (span) of $[S]$ defined as

$$
\operatorname{span}([S])=\left|S_{H H}\right|^{2}+\left|S_{V V}\right|^{2}+2\left|S_{H V}\right|^{2}
$$

is calculated and imaged in Fig. 4. Scattering contributions in all channels are summed and thus a better visualization is achieved. All manmade objects including the hidden trihedral C3 and the scatters within the gazebo area are better pronounced as hot spots. Furthermore, various bushes and grasses on surface vegetation become more visible within this image.

\subsection{Pauli Decomposition}

The direct use of $S_{H H}, S_{V V}$ and $S_{V H}$ elements, as seen above, is not much able to distinguish between different types of scattering mechanisms, especially between odd- and even-bounce. To overcome this limitation, we utilized decomposition techniques. First, Pauli decomposition was applied by constructing:

$$
k_{1}=\frac{S_{H H}+S_{V V}}{\sqrt{2}}, k_{2}=\frac{S_{H H}-S_{V V}}{\sqrt{2}}, k_{3}=\sqrt{2} S_{H V}
$$

components which correspond to the amount of contributions of odd-bounce, even-bounce and volume (or dihedral rotated $45^{\circ}$ ) scatterings, respectively. The RGB color composite image was then formed by assigning $\left|k_{1}\right|,\left|k_{2}\right|$ and $\left|k_{3}\right|$ as blue, red and green. Fig. 5 shows the resulted image. The following interpretations can be done by noting that scattering characteristics of a target are largely affected by its size, shape, pattern and orientation.

The ground cover is mainly represented in black due to specular reflections from smooth surfaces. Trees are shown to have mixture of colors with a wide distribution of reddish and greenish tones owing to dominant double-bounce and volumetric scatterings. As for the manmade targets, the rear part of the gazebo also exhibits complex scattering characteristics similar to those of trees. Note that $\mathrm{VH}$ (green) component is usually resulted from complicated structures and targets that have azimuthal orientations relative to radar LOS, both of which hold for this target. The front section, however, has magenta and purple tones implying varying compositions of odd- and even-bounce that may be emerged from the pavement at the bottom, two waste containers, reflector C2 and gazebo's structure pattern (see optical image in Fig. 2).

The perceived color for the reflectors lying on low-density grassses (C1, C4 and C5) is violet. This indicates the presence of even-bounce return in addition to stronger odd-bounce return which can be explained by non-ideal ground conditions. The hidden TCR (C3) is seen to have all of the three scattering mechanisms. Furthermore, the TCR near the gazebo (C2), is in magenta, implying a strong double-bounce scattering, which can be attributed to the nearby step-like pavement. As for the metal sticks, the horizontal cylinder T1 is displayed as red, due to dihedral structure formed by the ground plane. The vertically aligned stick T2 shows a combination of odd and double-bounce scattering, while the T3 and T4 both show predominant volume scattering components because of their azimuthal orientation angles. The results, in general, demonstrate that Pauli components provide more information about the underlying scattering process when compared to raw elements of $[S]$.

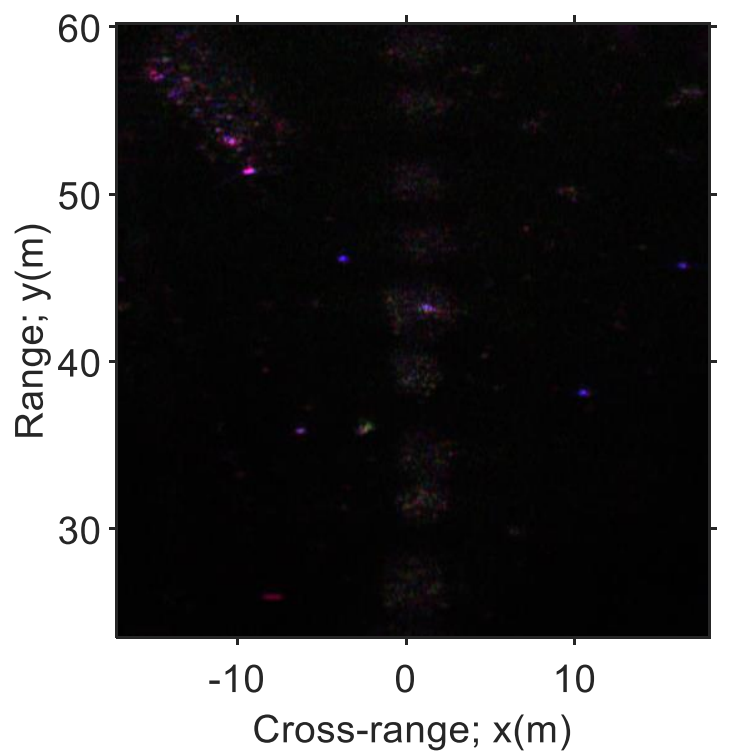

Figure 5. Color-coded composite image of the Pauli decomposition: blue, $\left|k_{1}\right|$; red, $\left|k_{2}\right|$; and green, $\left|k_{3}\right|$

\subsection{Krogager Decomposition}

Next, Krogager decomposition was utilized as a means of comparison with Pauli decomposition results. This approach, also called as the sphere, diplane, helix, (SDH) decomposition, coherently separates the symmetric $[S]$ matrix into three fundamental elements such as a sphere (plate), a diplane (dihedral) and a helix. This representation of $[S]$ in linear polarization basis can be written as (Krogager 1990) 


$$
[S]=e^{j \varphi}\left\{e^{j \varphi_{s}} k_{S}[S]_{\mathrm{S}}+k_{D}[S]_{\mathrm{D}(\theta)}+k_{H}[S]_{\mathrm{H}(\theta)}\right\}
$$

where, $\varphi$ is the absolute phase, phase $\varphi_{S}$ is the displacement of sphere to dihedral and helix components, $\theta$ is the orientation angle, and $[S]_{S}$, $[S]_{\mathrm{D}(\theta)}$ and $[S]_{\mathrm{H}(\theta)}$ denote the scattering from a sphere, a diplane and a helix with the corresponding weights $k_{S}, k_{D}$ and $k_{H}$, respectively. The approach offers an orientation invariant decomposition, but with a disadvantage that the diplane and helix matrices are not independent. But, the sphere and diplane, also the diplane and helix matrices are independent. Noting that helix scattering can be generated by two or more dihedrals, the absence of helix component, therefore, could be an indicative of pure even-bounce scatterer.

Fig. 6 shows the resulted composite RGB image obtained by discarding the phase terms and assigning the amplitudes of $k_{S}, k_{D}$ and $k_{H}$ as blue, red and green, respectively. The contributions of each of these scattering mechanisms are also presented individually in Fig. 7. We observe from these images that the scene has similar polarimetric scattering features with those of Pauli decomposition. For this case, green color in RGB image denotes helix scattering which appears typically in manmade structures or targets with complex shapes. The overall image is mostly dominated by reddish and blueish pixels, thereby implying a combination of odd- and even-bounce mechanisms. We also see that multiple-bounce returns from the gazebo and trees yield again a mixture of colors caused by their complex structures. The rear sections of the gazebo as well as the some tree sections show helix scattering denoting the presence of two or more even-bounce mechanisms within the resolution cells of these regions. Thus, compared to Pauli decomposition, pure even-bounce mechanisms can be more easily identified in Krogager decomposition by figuring out near red tones. In general, each of these coherent decompositions is shown to enable a simple and quite appropriate identification of elementary scattering mechanisms.

\subsection{Freeman-Durden Decomposition}

We have used two techniques for the incoherent analysis our polarimetric SAR data; FreemanDurden decomposition and eigenvalue/eigenvector decomposition. Each can be implemented through decomposition of the coherency matrix $\langle[T]\rangle$. For this reason, we first constructed the target scattering vector defined in Pauli basis as $\vec{k}_{P}=1 / \sqrt{2}\left[S_{H H}+\right.$ $\left.S_{V V}, S_{H H}-S_{V V}, 2 S_{V H}\right]^{T}$. The averaged coherency matrix $\langle[T]\rangle$, where $\langle\ldots\rangle$ stands for spatial averaging, was then obtained from the outer product of $\vec{k}_{P}$, followed by an averaging process over a $5 \times 5$ window.

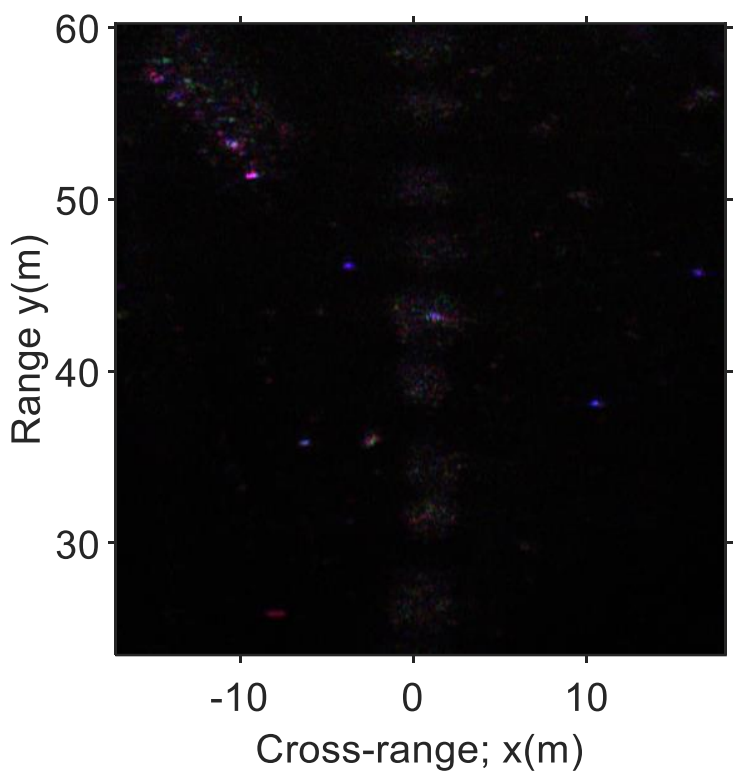

Figure 6. Color-coded composite image of the Krogager decomposition: blue, $\left|k_{S}\right|$; red, $\left|k_{D}\right|$; and green, $\left|k_{H}\right|$.

First, Freeman-Durden decomposition (Freeman and Durden 1998) was employed as an illustrative example of model-based decompositions. The technique decomposes the measured coherency matrix into a sum of three independent scattering models such that

$$
\langle[T]\rangle=f_{S}[T]_{S}+f_{D}[T]_{D}+f_{V}[T]_{V}
$$

where $[T]_{\mathrm{S}},[T]_{\mathrm{D}}$ and $[T]_{\mathrm{V}}$ denote respectively the scattering models for a surface, even- or doublebounce and volume scatters, with corresponding coefficients $f_{S}, f_{D}$ and $f_{H}$. These coefficients can be estimated from Eq. (4) from which the scattering power of each component, viz. $P_{S}, P_{D}$ and $P_{V}$ can be calculated. Finally, a RGB image can be derived to portray these power contributions in a single image.

Fig. 8 shows the composite RGB image obtained after applying Freeman-Durden decomposition on the study site's L-band data. Blue, red and green correspond to $\left|P_{S}\right|,\left|P_{D}\right|$ and $\left|P_{V}\right|$. These power contributions are also displayed separately in Fig. 9, to aid interpretation. The signatures of the canonical targets, i.e., CR1, CR4 CR5 and the vertical stick (T2) appear to be almost same as obtained previously. However the gazebo and tree canopy, in this case, are mainly shown in green color arisen from volume scattering. This is reasonable because of these targets' complicated geometric scattering structures. It is also worth pointing out that simple objects within the gazebo area, i.e., two waste containers and the trihedral C2 can now be clearly discerned and separated from the other pixels, with their dominant double-bounce scattering response. The trihedral within the trees (C3) has light green color which indicates the coexistence of odd-bounce and volume scatterings, as expected. On the other hand, the oriented sticks $\mathrm{T} 3$ and $\mathrm{T} 4$ are also represented in 
volume scattering category, revealing the technique's well-known limitation. More clearly, the decomposition is not able to discriminate between cross-polarized scattering caused by tilted manmade targets and cross-polarized scattering caused by vegetation (or tree) canopies. Nevertheless, it is apparent that Freeman-Durden decomposition provides a better characterization of the observed backscatter when compared to Pauli and Krogager decompositions.
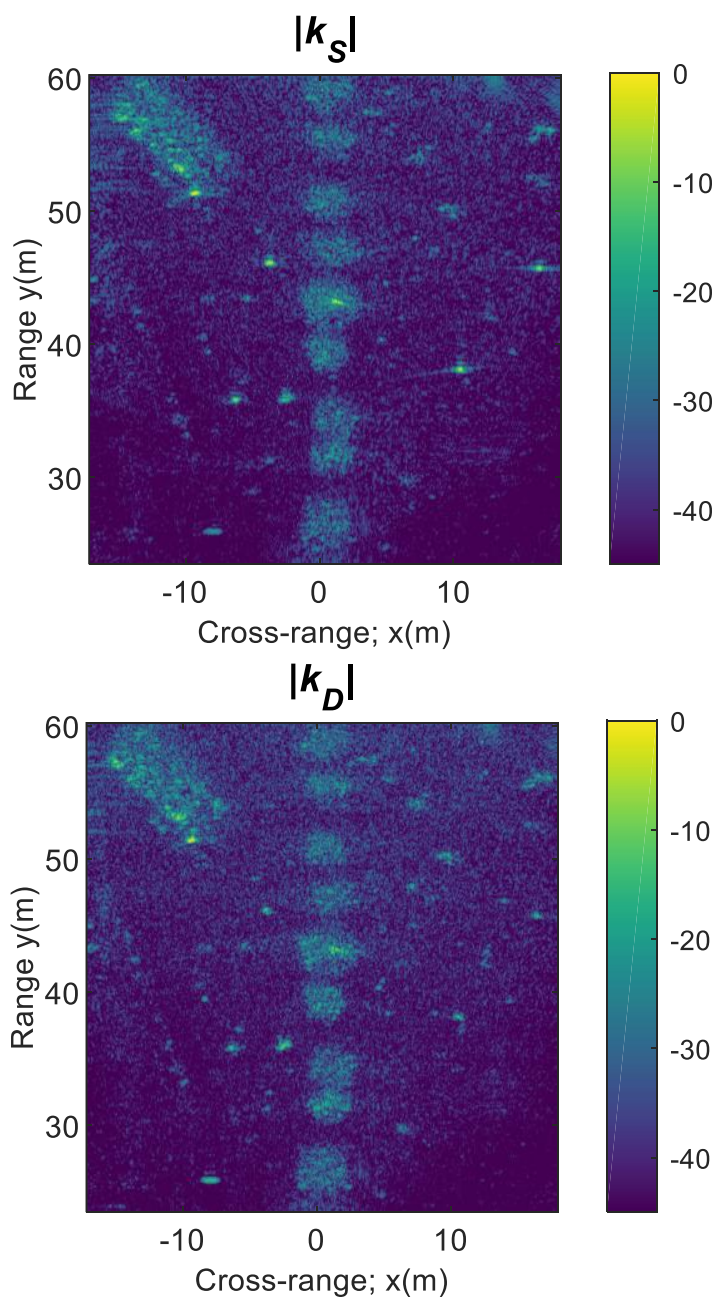

Cross-range; $x(m)$

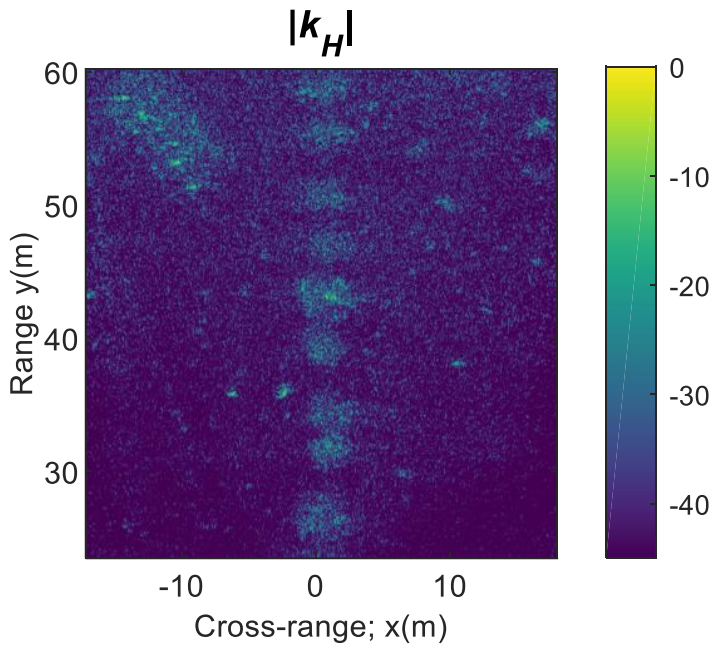

Figure 7. Amplitude images of Krogager decomposition components in a dB scale.

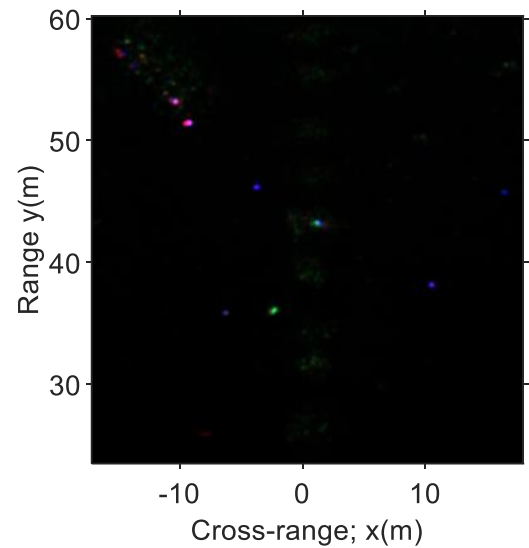

Figure 8. Freeman decomposition image with RGB color-coding: blue, $\left|P_{S}\right|$; red, $\left|P_{D}\right|$; and green, $\left|P_{H}\right|$.
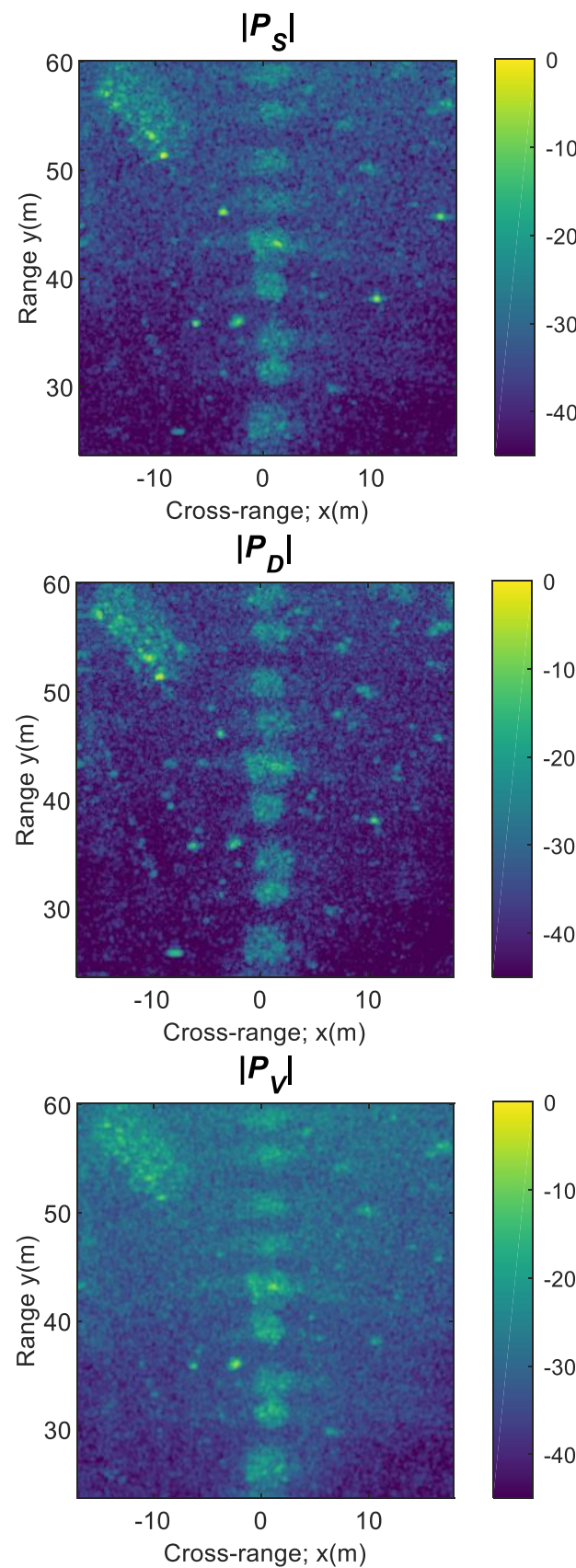

Figure 9. Power images of Freeman-Durden decomposition components in a dB scale 


\subsection{Eigenvalue/Eigenvector Decomposition}

Next, the widely-used eigenvalue/eigenvectorbased decomposition was utilized. For this purpose, the averaged coherency matrix $\langle[T]\rangle$ was diagonalised and expanded into incoherent sum of three independent coherency matrices $\left[T_{i}\right]$ as follows

$$
\langle[\mathrm{T}]\rangle=\left[T_{1}\right]+\left[T_{2}\right]+\left[T_{3}\right]=\sum_{i=1}^{3} \lambda_{i} \vec{e}_{i} \cdot \vec{e}_{i}^{+}
$$

where $\lambda_{1} \geq \lambda_{2} \geq \lambda_{3} \geq 0$ are the eigenvalues, $\vec{e}_{1}, \vec{e}_{2}$ and $\vec{e}_{3}$ are the unit eigenvectors and the superscript + denotes conjugate transpose operation. Each of the $\left[T_{i}\right]$ matrices represents a single deterministic scattering process, the strength and type of which are determined by the corresponding eigenvalue and eigenvector, respectively. From these primary parameters, secondary statistical parameters such as polarimetric entropy $(H)$, anisotropy $(A)$, alpha angle $(\alpha)$ and beta angle $(\beta)$ can be extracted and plotted for the interpretation of the information provided by the decomposition.

The top image in Fig. 10 shows the estimated entropy map of the investigated scene. Entropy is a measure of the degree of randomness of scattering which takes values between 0 and 1 . An entropy of 0 indicates a non-depolarizing (deterministic) scattering while 1 indicates a fully-depolarizing (random) scattering. We observe that entropy is high in most of the tree regions, as expected. In addition, high entropy also occurs over the vegetated areas that are more distant from the radar whereas the nearer areas have low entropy. This can become evident when considering the Rayleigh roughness criterion for surface scattering. More specifically, the illumination of farther targets with lower incidence angles (see Fig. 1) gives rise to more penetration of electromagnetic waves into scatter ensemble, thereby resulting in a depolarized scattering. Concerning the manmade targets, the rear part of the gazebo has high entropy because of random vector scattering from structurally complex targets. The front part, on the other hand, has low entropy, as a result of coherent reflections. Besides, the two waste containers and the trihedral C2 within that area, have nearly minimum $H$ values, so are showing a nondepolarizing mechanism. This is also observed to be true for the other reflectors, except the concealed one. Lastly, the sticks exhibit weakly depolarizing behavior as evident from low to moderate $H$ values.

The type of scattering process, as mentioned, is associated with the eigenvector information. The mean-alpha angle $\bar{\alpha}$ is an average representation of this information and mainly used for the prediction of the dominant scattering mechanism present in the target. It ranges from $0^{\circ}$ to $90^{\circ}$ with values; in general, $0^{\circ} \leq \bar{\alpha} \leq 30^{\circ}, 40^{\circ} \leq \bar{\alpha} \leq 50^{\circ}$ and $60^{\circ} \leq \bar{\alpha} \leq$ $90^{\circ}$ are regarded as surface, dipole and dihedral scatterings, respectively. The middle image in Fig. 10 shows the spatial distribution of $\bar{\alpha}$ values for the investigated scene. Targets that cause doublebounce mechanisms can be recognized as greenish to yellowish colors. Various parts of the trees and gazebo, and the horizontal cylinder present such expected mechanism. The tilted stick (T3) with $\bar{\alpha}$ around $65^{\circ}$, however, also has double-bounce scattering, rather than dipole scattering. Note that $\alpha$ parameter is independent from the orientation of the target about the radar LOS. Thus, these doublebounce returns are supposed to be originated from the bottom of the target, where a stone was used as a support material. Finally, the terrain with low vegetation and the reflectors have $\bar{\alpha}$ values below $30^{\circ}$ indicating a dominance of anisotropic surface or single-bounce mechanism.

Another parameter that can be obtained is the $\beta$ angle which describes the orientation of a target about the LOS. The bottom image in Fig. 10 shows this map of the scene whereby all the targets that have orientations in azimuth direction, namely, the two $45^{\circ}$ oriented sticks, the gazebo structure, the trees and various bushes can be readily distinguished.
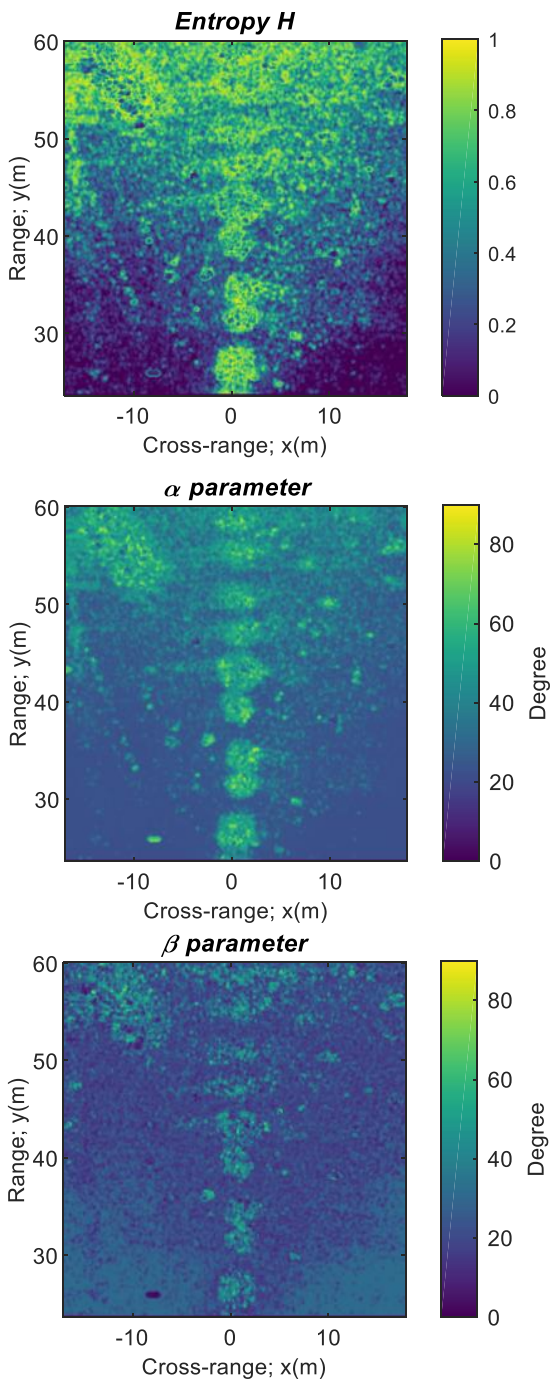

Figure 10. Entropy, $\bar{\alpha}$ and $\bar{\beta}$ angle maps for the test site, obtained after application of eigenvalue/eigenvector decomposition 


\section{6 $H / \bar{\alpha}$ classification}

The entropy $(H)$ and mean alpha $(\bar{\alpha})$ values can be used in pairs for classification of random scattering mechanisms. The $H / \bar{\alpha}$ classification scheme proposed in (Cloude and Pottier 1997) for Lband is based on the subdivision of the $H / \bar{\alpha}$ plane into 9 scattering classes (8 usable) whose partitioning and descriptions are illustrated in Fig. 11(a). The result of applying this classification scheme is shown in Fig. 11(b) where the assigned color of each class is also given on the right.

Pixels belonging to the trees, plants and gazebo are mostly classified into Z3. This reflects moderately random multiple-bounce mechanisms mainly associated with trunk-ground interactions. Besides, there are also pixels classified as Z4 which indicates medium entropy dipole-like scattering due to secondary scattering processes. Both of these mechanisms are also seen for the gazebo area. However, the waste containers and the trihedral can be distinguished from this structure, with lowentropy dipole (Z7) and surface-like (Z8) scatterings. The TCR targets are correctly classified as Z8. Even the hidden TCR can be discerned from its blue color. Concerning the other targets, the oriented stick T3 is recognized to have each type scattering classes of Z3, $\mathrm{Z6}$ and Z7, which can be again attributed to its base support. Lastly, the horizontal cylinder as well as some plants along the pave road are classified as Z6 showing a deterministic dihedral scattering.

\section{CONCLUSION}

We presented a proof-of-concept type study for the employment of polarimetric GB-SAR imaging technology. An experimental assessment of the polarimetric L-band backscattering of a typical terrain and man-made targets was made through GB-SAR data. The measurement set-up and the polarimetric analysis techniques were explained. The coherent and incoherent decompositions of backscattering data provided satisfactorily efficient means to interpret the dominant scattering mechanisms occurring within the scene.

\section{ACKNOWLEDMENT}

The authors are grateful to Mersin Technology Development Zone (Technoscope) for providing laboratory facilities for the conducted experiments.

\section{REFERENCE}

Alberga V, Krogager E, Chandra M \& Wanielik G (2004). Potential of coherent decompositions in SAR polarimetry and interferometry. 2004 IEEE International Geoscience and Remote Sensing Symposium, Anchorage, AK, USA.

Albinet C, Borderies P, Koleck T, Rocca F, Tebaldini S, Villard L, Toan T L, Hamadi A \& Minh D H T (2012). TropiSCAT: A ground based
The authors are thankful to Dr. Betul Yilmaz, Hakan Isıker and Serhat Gokkan, for their valuable help during the experiments.

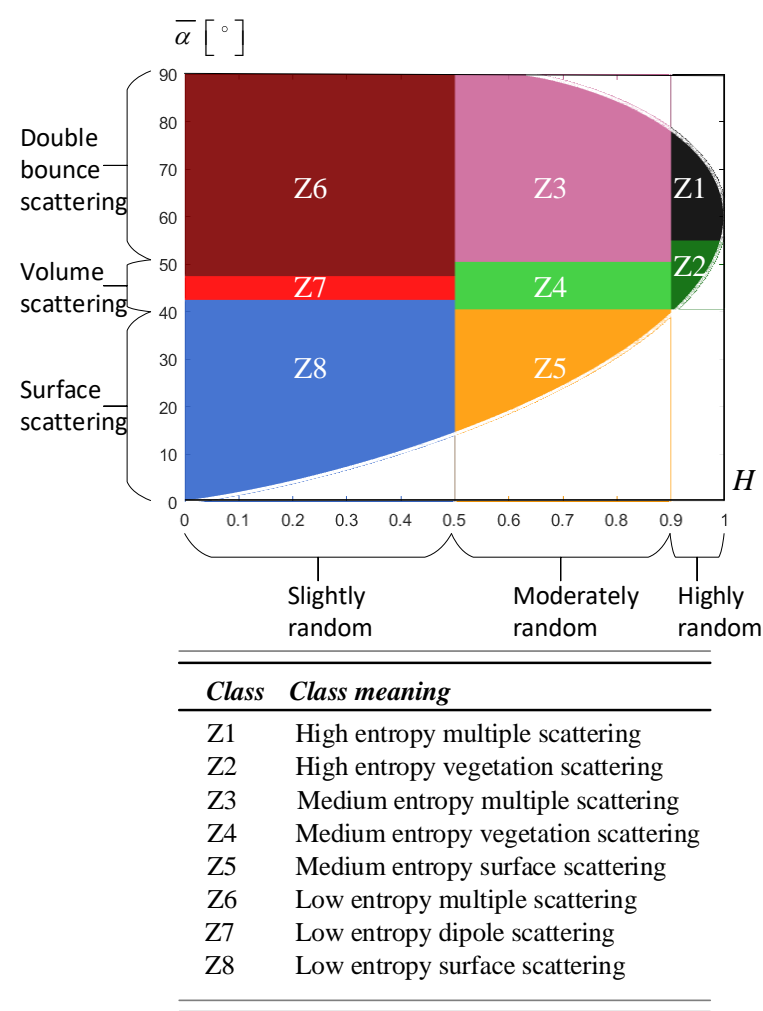

(a)

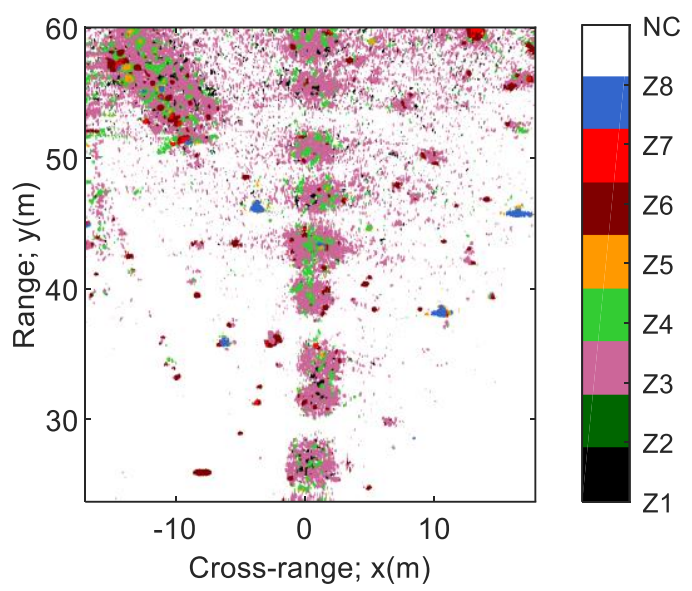

(b)

Figure 11. (a) $H / \bar{\alpha}$ plane with classes (Z1-Z8) and their partitioning and descriptions. (b) $H / \bar{\alpha}$ classification result for the test scene (NC means notclassified)

polarimetric scatterometer experiment in tropical forests. IEEE Journal of Selected Topics in Applied Earth Observations and Remote Sensing (JSTARS), 5(3), 1060-1066.

Baffelli S, Frey O, Werner C \& Hajnsek I (2018). Polarimetric calibration of the $\mathrm{Ku}$ Band advanced polarimetric radar interferometer 
(KAPRI). IEEE Transactions on Geoscience and Remote Sensing, 56(4), 2295-2311.

Brown S C M, Quegan S, Morrison K, Bennett J C \& Cookmartin G (2003). High-resolution measurements of scattering in wheat canopiesimplications for crop parameter retrieval. IEEE Transactions on Geoscience and Remote Sensing, 41(7), 1602-1610.

Chen S W, Li Y Z, Wang X S, Xiao S P \& Sato M (2014). Modeling and interpretation of scattering mechanisms in polarimetric synthetic aperture radar: Advances and perspectives. IEEE Signal Processing Magazine, 31, 79-89.

Chen S W, Wang X S, Xiao S P \& Sato M (2018). Advanced polarimetric target decomposition. In: Target scattering mechanism in polarimetric synthetic aperture radar. Singapore: Springer.

Cho B L, Kong Y K, Park H G \& Kim Y S (2006). Automobile-based SAR/InSAR system for ground experiments. IEEE Geoscience and Remote Sensing Letters, 3(3), 401-405.

Cloude S R \& Pottier E (1996). A review of target decomposition theorems in radar polarimetry. IEEE Transactions on Geoscience and Remote Sensing, 34(2), $498-518$.

Cloude S R \& Pottier E (1997). An entropy based classification scheme for land applications of polarimetric SAR. IEEE Transactions on Geoscience and Remote Sensing, 35(1), 68-78.

Cloude S R (2010). Polarisation application in remote sensing. Oxford: Oxford Univ. Press.

Cuenca L M (2017). Contribution to ground-based and UAV SAR systems for Earth observation. Ph. D. dissertation, Universitat Politecnica de Catalunya, Barcelona, Spain.

Demirci S, Yilmaz B, Isiker H, Gokkan S \& Ozdemir C (2019). Characterization of natural and manmade targets from L-band ground-based polarimetric synthetic aperture radar data. Journal of Applied Remote Sensing, 13(4), doi: 10.1117/1. JRS.13.044512.

Freeman A \& Durden S L (1998). A three-component scattering model for polarimetric SAR data. IEEE Transactions on Geoscience and Remote Sensing, 36(3), 963-973.

Gonzalez-Partida J T, Almorox-Gonzalez P, BurgosGarcia M \& Dorta-Naranjo B P (2008). SAR system for UAV operation with motion error compensation beyond the resolution cell. Sensors, 8(5), 3384-3405. DOI: $10.3390 / \mathrm{s} 8053384$

Iglesias R, Aguasca A, Fabregas X, Mallorqui J J, Monells D \& Lopez C (2015a). Ground-based polarimetric SAR interferometry for the monitoring of terrain displacement phenomena-Part I: Theoretical description. IEEE Journal of Selected Topics in Applied Earth Observations and Remote Sensing, 8(3), 980993.

Iglesias R, Aguasca A, Fabregas X, Mallorqui J J, Monells D \& Lopez C (2015b). Ground-based polarimetric SAR interferometry for the monitoring of terrain displacement phenomena-Part II: applications. IEEE Journal of Selected Topics in Applied Earth Observations and Remote Sensing. 8(3), 9941007.

Kang M K, Kim K E, Lee H, Cho S J \& Lee J H (2009). Preliminary results of polarimetric characteristics for C-band quad-polarization GB-SAR images using $\mathrm{H} / \mathrm{A} / \mathrm{alph}$ a polarimetric decomposition theorem. Korean Journal of Remote Sensing, 25(6), 531-546.

Krogager E (1990). New decomposition of the radar target scattering matrix. Electronics Letters, 26, 1525-1527.

Lee H, Cho S J, Sung N H \& Kim J H (2007). Development of a GB-SAR (II): Focusing algorithms. Korean Journal of Remote Sensing, 23(4), 247-256.

Lee H, Ji Y \& Han H (2016). Experiments on a groundbased tomographic synthetic aperture radar. Remote Sensing, 8(8), 1-11. DOI: $10.3390 / \mathrm{rs} 8080667$

Lee H, Lee J -H, Kim K -E, Sung N -H \& Cho S -J (2014). Development of a truck-mounted arcscanning synthetic aperture radar. IEEE Transaction on Geoscience and Remote Sensing, 52(5), 2773-2779.

Lee J \& Pottier E (2009). Polarimetric radar imaging: From basics to applications. Boca Raton: Taylor \& Francis.

Lim K S \& Koo V C (2008). Design and construction of wideband VNA ground-based radar system with real and synthetic aperture measurement capabilities. Progress in Electromagnetics Research, PIER, 86, 259-275.

Minh D H T, Tebaldini S, Rocca F, Toan T L, Borderies P, Koleck T, Albinet C, Hamadi A \& Villard L (2014). Vertical structure of P-Band temporal decorrelation at the Paracou forest: Results from TropiScat. IEEE Geoscience and Remote Sensing Letters, 11(8), 1438-1442.

Moreira A, Prats-Iraola P, Younis M, Krieger G, Hajnsek I \& Papathanassiou K (2013). A tutorial on synthetic aperture radar. IEEE Geoscience and Remote Sensing Magazine, 1(1), 6-43. DOI: 10.1109/MGRS.2013.2248301

Ouchi K (2013). Recent trend and advance of synthetic aperture radar with selected topics. Remote Sensing, 5(2), 716-807. DOI: 10.3390/rs5020716

Ozdemir C, Demirci S, Yigit E \& Yilmaz B (2014). A review on migration methods in B-Scan ground penetrating radar imaging. Mathematical Problems in Engineering, 1-16. DOI: $10.1155 / 2014 / 280738$

Penner J F \& Long D G (2017). Ground-based 3D radar imaging of trees using a $2 \mathrm{D}$ synthetic aperture. Electronics, 6(11), 1-13. DOI: 10.3390/electronics6010011

Pipia L (2009). Polarimetric differential SAR interferometry with ground-based sensors. 
Ph.D. dissertation, Universitat Politecnica de Catalunya, Barcelona, Spain.

Pipia L, Fabregas X, Aguasca A \& Lopez-Martinez C (2013). Polarimetric temporal analysis of urban environments with a ground-based SAR. IEEE Transactions on Geoscience and Remote Sensing, 51(4), 2343-2360.

Sevgen S C (2019). Airborne LIDAR data classification in complex urban area using random forest: A case study of Bergama, Turkey. International Journal of Engineering and Geosciences, 4(1), 45-51. DOI: 10.26833/ijeg.440828

The MathWorks, Inc. MATLAB, Release 2018. Natick, Massachusetts, United States.

Van Zyl J J \& Kim Y (2011). Synthetic aperture radar polarimetry. Hoboken, NJ: John Wiley \& Sons, Inc.

Xing S, Li Y, Dai D \& Wang X (2013). Threedimensional reconstruction of man-made objects using polarimetric tomographic SAR. IEEE Transactions on Geoscience and Remote Sensing, 51(6), 3694-3705.
Yilmaz A \& Erdogan M (2018). Designing high resolution countrywide DEM for Turkey. International Journal of Engineering and Geosciences, 3(3), 98-107. DOI: 10.26833/ijeg.384822

Yilmaz M \& Uysal M (2017). Comparing uniform and random data reduction methods for DTM accuracy. International Journal of Engineering and Geosciences, 2(1), 9-16. DOI: 10.26833/ijeg. 286003

Zhou Z S (2003). Application of a ground-based polarimetric SAR system for environmental study. Ph.D. dissertation, The Graduate School of Engineering, Tohoku University, Sendai, Japan.

Zhou Z S (2004). Development of a ground-based polarimetric broadband SAR system for noninvasive ground-truth validation in vegetation monitoring. IEEE Transactions on Geoscience and Remote Sensing, 42(9), 18031810. 